\title{
Podoplanin-positive Cancer-associated Stromal Fibroblasts in Primary Tumor and Synchronous Lymph Node Metastases of HER2-overexpressing Breast Carcinomas
}

\author{
JOANNA NIEMIEC ${ }^{1}$, AGNIESZKA ADAMCZYK ${ }^{1}$, AGNIESZKA HARAZIN-LECHOWSKA ${ }^{1}$, \\ ALEKSANDRA AMBICKA ${ }^{1}$, ALEKSANDRA GRELA-WOJEWODA ${ }^{2}$, KAJA MAJCHRZYK ${ }^{1}$, \\ ANNA KRUCZAK ${ }^{1}$, BEATA SAS-KORCZYŃSKA ${ }^{3}$ and JANUSZ RYŚ ${ }^{1}$ \\ Departments of ${ }^{1}$ Tumour Pathology, ${ }^{2}$ Systemic and Generalized Malignancies, and \\ ${ }^{3}$ The Oncology Clinic, Maria Skłodowska-Curie Memorial Center and Institute of Oncology, \\ Cracow Branch, Cracow, Poland
}

\begin{abstract}
We compared the status of stromal podoplaninpositive cancer-associated fibroblasts ( $p p C A F s$ ) between primary tumors and paired synchronous lymph node metastases (LNMs) and analyzed the prognostic significance of tumoral ppCAFs in 203 patients with human epidermal growth factor receptor 2-positive breast carcinoma. ppCAFs were found in 167/203 and in 35/87 tumors and LNM, respectively. ppCAFs were most frequently found in tumors and corresponding $\operatorname{LNM}(n=52,59.8 \% ; p=0.001)$. However, for all LNMs $(n=12)$ without ppCAFs, their paired tumors also lacked ppCAFs. In both tumors and LNMs, ppCAFs were $\alpha$-smooth muscle actin-positive and cluster of differentiation 21 protein-negative, suggesting them not to be resident lymph node cells. Moreover, in our series, the presence of ppCAFs in tumors was borderline related to poor disease-free survival $(p=0.058)$. These results speak in favor of a hypothesis suggesting ppCAFs accompany metastatic cancer cells migrating from tumor to LNMS.
\end{abstract}

Numerous studies have shown that cancer development and metastasis formation are complex processes which involve not only cancer cells but also their interaction with selected components of tumor stroma. According to the seed and soil

This article is freely accessible online.

Correspondence to: Joanna Niemiec, Department of Tumour Pathology, Maria Sklodowska-Curie Memorial Cancer Centre and Institute of Oncology, Cracow Branch, ul. Garncarska 11, 31-115 Cracow, Poland. Tel: +48 12 6348415, e-mail: joanna@eikon.pl, z5niemie@cyfronet.pl

Key Words: Podoplanin-positive cancer-associated fibroblasts, primary tumor, lymph node metastases, prognostic significance. hypothesis, a permissive microenvironment (soil) facilitates growth of metastatic cancer cells (seed) in secondary sites. It was shown that stromal components (including cancerassociated fibroblasts, CAFs) can travel together with metastatic cells from the primary to secondary site. Moreover, co-traveling stromal cells can increase the viability of circulating metastatic cancer cells and provide them with an early growth advantage (1). It was also shown that CAFs promote tumor invasion by exerting a physical force on cancer cells, enabling them to escape from the primary site (2). The identification of CAFs in peripheral blood from patients with metastatic breast cancer confirms the hypothesis suggesting co-travel of stromal and cancer cells during formation of metastases (3).

In vitro studies and research based on animal models suggest a very important role of the cancer stroma in tumor progression $(1,4)$. The above phenomena were confirmed by studies in which the following stroma-related parameters were found to be of prognostic significance: (i) tumor-tostroma ratio (5-7); (ii) the type of tumor stroma (fibroblastdominant $v s$. lymphatic-dominant $v s$. collagen-dominant) (810); (iii) the presence of podoplanin-positive CAFs (ppCAFs) (11-14). Furthermore, it was suggested that CAFs might contribute to: (i) tumor initiation; (ii) proliferation, migration and invasiveness of cancer cells; (iii) tumor angiogenesis/lymphangiogenesis; (iv) tumor-induced inflammation; (v) metabolic reprogramming of the tumor microenvironment; and (vi) therapeutic resistance (4). Among many CAF-related proteins, podoplanin is one of the most intensively studied $(8,9,15-17)$. Among breast cancer immunophenotypes, the human epidermal growth factor receptor 2 (HER2)-overexpressing subtype is the one in which ppCAFs were found to be relatively frequent (11).

This prompted us to analyze: (i) the status of ppCAFs in primary tumor and paired synchronous lymph node metastases (LNMs); (ii) relation between ppCAFs in tumor 
and clinical/biological parameters; (iii) immunophenotype of ppCAFs [markers of follicular dendritic cells (FDCs) or fibroblastic reticular cells (FRCs): $\alpha$-smooth muscle actin ( $\alpha$ SMA), cluster of differentiation 21 (CD21), podoplanin]; and (iv) the prognostic significance of tumoral ppCAFs, in a group of 203 patients with HER2-positive breast carcinoma who were treated with trastuzumab in the adjuvant setting.

\section{Materials and Methods}

Patients. We included 203 patients with HER2-overexpressing breast carcinoma $(\mathrm{pT} \geq 1, \mathrm{pN} \geq 0, \mathrm{M} 0)$, treated with trastuzumab and chemotherapy in an adjuvant setting (112 women received anthracyclines, 90 anthracyclines and taxanes, while one patient did not agree to chemotherapy). All patients underwent radical local therapy comprising surgery: 101 underwent Madden's mastectomy, 75 breast-conserving surgery (BCS), nine mastectomy and sentinel lymph node dissection, and $18 \mathrm{BCS}$ and lymphadenectomy (postoperative radiotherapy was applied for 168 patients) at the Maria SklodowskaCurie Memorial Cancer Centre and Institute of Oncology, Cracow Branch, Poland, between 2008 and 2013. One hundred and eighteen patients with estrogen receptor (ER) or progesterone receptor (PR)positive carcinomas (Table I) received hormonal therapy.

In our series, the median survival was 45 (range 14-99) months. During follow-up, metastases were observed in $18 / 203$ patients. In five out of 18 patients with progressive carcinomas, both metastasis and local recurrence were found.

This was a retrospective study utilizing archived tissues, with no direct patient contact, no modification of diagnostic or treatment procedures and no personal patient data revealed, therefore, no specific consent was needed. Study approval was given by the Ethical Committee at the Regional Medical Chamber in Cracow (decision from 4th December 2013).

Materials. Tumor specimens from formalin-fixed paraffin-embedded tissues were re-examined independently by two pathologists (A.HL., A.A.) to confirm histological diagnosis and tumor grade and to assess tissue amount and quality needed for this study.

Immunohistochemical staining. Podoplanin, CD34, aSMA and CD21 expression was visualized immunohistochemically on formalin-fixed paraffin-embedded tissue sections mounted on SuperFrost slides (Menzel-Gläser, Braunschweig, Germany). Antigen retrieval (Target Retrieval Solution: $\mathrm{pH} 6.1 ; 96^{\circ} \mathrm{C}$ for $50 \mathrm{~min}$; DakoCytomation Denmark A/S, Glostrup, Denmark) was applied for CD21, podoplanin and CD34. Quenching the activity of endogenous peroxidases was performed in $0.3 \% \mathrm{H}_{2} \mathrm{O}_{2}$ in $100 \%$ methanol (30 min incubation; room temperature), and blocking of nonspecific antibody binding in UltraVision Protein Block (5 min; room temperature; Thermo Scientific, Fremont, CA, USA). Next, after incubation with primary antibody (Table II) and BrightVision (30 min; room temperature, Immunologic, Duiven, the Netherlands), peroxidase substrates [3,3'-diaminobenzidine (DAB), and VECTOR VIP from Peroxidase (HRP) Substrate Kits; Vector Laboratories, Burlingame, CA, USA] were applied to visualize detected antigens. DAB (brown color) was used for visualization of podoplanin in double-stain proceure as well as for visualization of other markers (Figures 1-4), while VIP (violet color) for visualization of CD34 in double-stain procedure (Figures 1, 2d-f, 4a and d). Finally, slides were counterstained with Mayer's hematoxylin.
Table I. Relationship between presence of podoplanin-positive cancerassociated fibroblasts (ppCAFs) in tumor and clinical/histological parameters.

\begin{tabular}{|c|c|c|c|c|}
\hline \multirow[b]{2}{*}{ Parameter } & \multirow[b]{2}{*}{$\mathrm{n}$} & \multicolumn{3}{|c|}{ ppCAFs in tumor } \\
\hline & & Not detected & Present & $p$-Value* \\
\hline \multicolumn{5}{|l|}{ Age, years } \\
\hline- & 203 & $53.1 \pm 10.5$ & $55.3 \pm 10.2$ & 0.249 \\
\hline \multicolumn{5}{|l|}{$\mathrm{pT}, \mathrm{n}$} \\
\hline 1 & 84 & 18 & 66 & \\
\hline $2+3$ & 112 & 18 & 94 & 0.357 \\
\hline \multicolumn{5}{|l|}{$\mathrm{pN}, \mathrm{n}$} \\
\hline 0 & 99 & 25 & 74 & \\
\hline+ & 103 & 11 & 92 & 0.009 \\
\hline \multicolumn{5}{|l|}{ Grade, $\mathrm{n}$} \\
\hline 2 & 66 & 14 & 52 & \\
\hline 3 & 132 & 20 & 112 & 0.319 \\
\hline \multicolumn{5}{|l|}{ ER/PR, n } \\
\hline Negative & 83 & 6 & 77 & \\
\hline Positive & 118 & 30 & 88 & 0.001 \\
\hline \multicolumn{5}{|l|}{ Ki-67 LI, \% } \\
\hline- & 198 & $38.0 \pm 15.3$ & $40.8 \pm 14.4$ & 0.309 \\
\hline
\end{tabular}

$\mathrm{n}$ : Number of cases; ER: estrogen receptor; PR: progesterone receptor; LI: labelling index. *One-way ANOVA (mean \pm SD) for relationship between categorical and continuous variables, from Fisher test for relationship between two categorical variables.

Immunohistochemical evaluation. Podoplanin-positive stromal CAFs (ppCAFs) were defined as spindle-shaped stromal cells without lymphatic vessel characteristics, presenting indistinct cell borders, with podoplanin expression (weaker, equal to or stronger than podoplanin expression in lymphatic endothelium) (Figure $1 \mathrm{a}-\mathrm{c}, \mathrm{f}, \mathrm{g}$, asterisk). The presence of ppCAFs was confirmed when the aforementioned stromal components were found in $>10 \%$ of cancer stroma. Lymphatic vessels were defined as podoplanin-stained structures with lymphatic vessel characteristics, clearly distinguishable from other tissue structures/cells (Figure 1g, black arrow) and from CD34-stained blood vessels (Figure 1a-e and g, arrowhead).

Methods applied for assessment of ER, PR, HER2 and Ki67 expression were described in our previous report (18).

Statistical analysis. STATISTICA v.12 software (StatSoft, Inc. Tulsa, OK, USA) was used for all calculations. $p$-Values less than 0.05 were considered significant. Relations between continuous and categorical variables were studied using one-way ANOVA, and assessment of independence between two categorical variables using Fisher test $(2 \times 2)$. Disease-free survival (DFS) was defined as the time from surgery to clinical or radiological relapse of the tumor. The probability of survival was calculated using the Kaplan-Meier method.

\section{Results}

ppCAFs in tumor and paired synchronous LNMs - analysis of 87 cases. In primary tumors, ppCAFs were found in $167 / 203(82.3 \%)$ cases, and in 35/87 (40.2\%) of LNM cases (Figure 1a-f). The presence of ppCAFs in tumor stroma was 
Table II. Immunohistochemical procedures.

\begin{tabular}{ll}
\hline Reaction with primary antibody & Reagent manufacturer \\
\hline Antibody to podoplanin (clone D2-40): dilution $1: 100 ; 37^{\circ} \mathrm{C} ; 1 \mathrm{~h}$ & CellMarque, Rocklin, CA, USA \\
Antibody to CD34 (clone QBEnd 10): dilution $1: 50 ; 4^{\circ} \mathrm{C} ;$ overnight) & DakoCytomation Denmark A/S, Glostrup, Denmark \\
Antibody to SMA (clone NCL-SMA): dilution $1: 50 ; 4^{\circ} \mathrm{C}$; overnight & Leica Biosystems, Nussloch GmbH, Germany \\
Antibody to CD21 (clone 2G9): dilution $1: 50 ; 4^{\circ} \mathrm{C} ;$ overnight & CellMarque, Rocklin, CA, USA \\
\hline
\end{tabular}

found significantly more frequently in patients with nodepositive disease $(p=0.009)$ and in carcinomas characterized by ER/PR negativity ( $p=0.001)$ (Table I).

Most frequently (52 out of 87 cases), ppCAFs were present both in primary tumor and in synchronous LNMs $(p<0.001)$ (Table III). However, in 23 cases, ppCAFs were present only in primary tumors and not in paired nodal sections (Table III). In the case of 12 tumors lacking ppCAFs, their paired LNMs presented no podoplanin expression in CAFs (Table III).

Survival analysis. In the studied group, the presence of ppCAFs was statistically insignificantly related to poorer patient survival $(p=0.058)$. None of the 36 patients with carcinomas devoid of ppCAFs developed distant metastases, while all 18 cases with metastases were observed in the group of individuals with tumor ppCAFs. In the abovementioned group, the Kaplan-Meier metastasis-free survival rate was $85.8 \%$.

Immunophenotype of ppCAFs in tumor and paired synchronous LNMs. In normal breast tissue, podoplanin expression was found in the basal layer of normal ducts and lobules (Figure 2d; black arrow) and in lymphatic vessels (Figure 2d; white asterisk). We did not find any CD21 expression in normal breast tissue. $\alpha$ SMA expression was detected in myoepithelial cells of normal ducts and lobules (Figure 2a, black arrow) and muscle layer of normal blood vessels (Figure 2a, white asterisk). Fibroblasts of normal breast connective tissue were podoplanin and $\alpha$ SMA immunonegative (Figure 2a and $\mathrm{d}$ ). On the other hand, in breast carcinomas (Figure $2 \mathrm{~b}, \mathrm{c}, \mathrm{e}, \mathrm{f}$ ), all CAFs were $\alpha$ SMA-positive (Figure $2 b$ and c; black arrowhead), but only some of them showed additional podoplanin positivity (Figure 2f: black arrowhead, but not e).

In order to determine the origin of ppCAFs in LNMs, we tested combination of markers specific for FDCs and FRCs, i.e. podoplanin, $\mathrm{CD} 21$ and $\alpha \mathrm{SMA}$ in lymph nodes without metastases (Figure 3a-c), primary tumor (Figure 4a-c), and synchronous LNMs (Figure 4d-f).

In lymph nodes without metastases, podoplanin expression was found in endothelial cells of lymphatic sinuses (Figure
Table III. Relationship between the presence of podoplanin-positive cancer-associated fibroblasts ( $p$ CAFs) in primary tumors and lymph node metastases $(p<0.001)$.

\begin{tabular}{lcc}
\hline & \multicolumn{2}{c}{ ppCAFs in lymph node metastases } \\
\cline { 2 - 3 } & Not detected, $\mathrm{n}$ & Present, $\mathrm{n}$ \\
\hline ppCAFs in primary tumor & & \\
Not detected, $\mathrm{n}$ & 12 & 0 \\
Present, $\mathrm{n}$ & 23 & 52 \\
\hline
\end{tabular}

3a, black arrow). Moreover, in normal lymph nodes, we confirmed the following immunophenotype of FDCs: podoplanin ${ }^{+} / \mathrm{CD} 21^{+} / \alpha \mathrm{SMA}^{-}$(Figure $3 \mathrm{a}, \mathrm{b}$, and $\mathrm{c}$, respectively, white asterisk), as well as the following immunophenotype of FRCs: podoplanin ${ }^{-} / \mathrm{CD} 21^{-} / \alpha \mathrm{SMA}^{+}$ (Figure $3 \mathrm{a}, \mathrm{b}$ and $\mathrm{c}$, respectively; $\alpha$ SMA immunopositivity identified with black arrow in c).

In order to deduce the origin of ppCAFs in LNMs, we then compared staining pattern of podoplanin, $\alpha$ SMA and CD21 on serial sections and in the same microscopic fields of tumors (Figure 4a, b, c) and of LNMs (Figure 4d, e and f). We excluded the possibility that ppCAFs in LNMs are FDCs because the immunophenotype of FDCs was podoplanin ${ }^{+} /$ $\mathrm{CD} 1^{+} / \alpha \mathrm{SMA}^{-}$(Figure $3 \mathrm{a}, \mathrm{b}$ and $\mathrm{c}$, respectively, white asterisk), while that of ppCAFs was $\mathrm{CD} 21^{-} / \alpha \mathrm{SMA}^{+}$(Figure $4 \mathrm{~b}$, e and c, f; $\alpha$ SMA identified with black arrow in $\mathrm{c}$ and $\mathrm{f}$ ). Moreover we excluded the possibility that ppCAFs in LNM are FRCs because the immunophenotype of FRCs was podoplanin ${ }^{-} / \mathrm{CD} 21^{-} / \alpha \mathrm{SMA}^{+}$(Figure $3 \mathrm{a}, \mathrm{b}$ and c; $\alpha \mathrm{SMA}$ immunopositivity identified with black arrow in c), while ppCAFs showed podoplanin expression (Figure $4 \mathrm{a}$ and $\mathrm{d}$ white asterisk).

\section{Discussion}

The results of the present study, obtained from a group of trastuzumab-treated patients with HER2-overexpressing breast cancer, confirmed the relationship between the presence of ppCAFs and ER/PR immunonegativity, and 

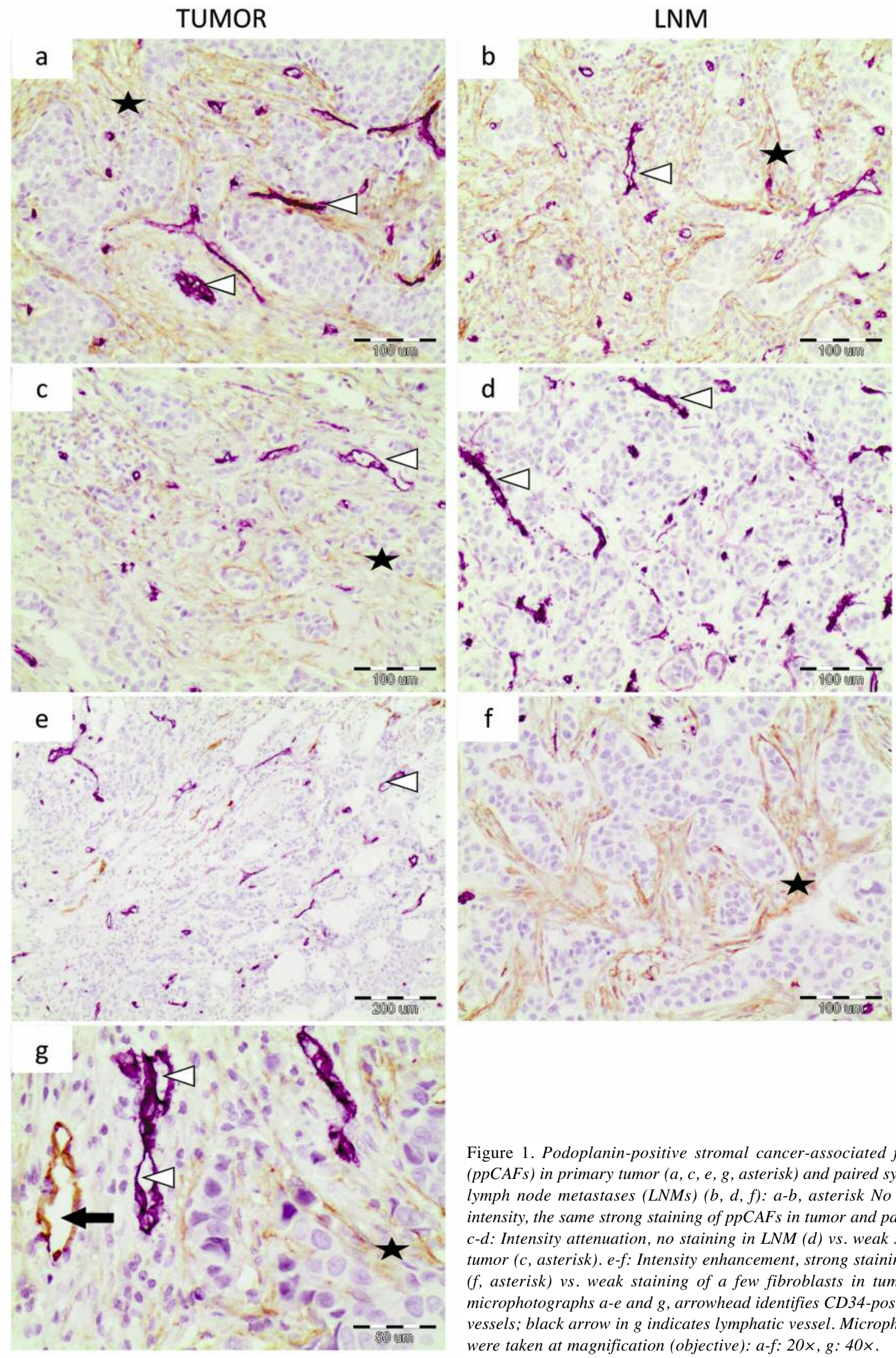

Figure 1. Podoplanin-positive stromal cancer-associated fibroblasts (ppCAFs) in primary tumor ( $a, c, e, g$, asterisk) and paired synchronous lymph node metastases (LNMs) $(b, d, f): a-b$, asterisk No change in intensity, the same strong staining of ppCAFs in tumor and paired LNM. $c$-d: Intensity attenuation, no staining in LNM (d) vs. weak staining in tumor (c, asterisk). e-f: Intensity enhancement, strong staining in LNM (f, asterisk) vs. weak staining of a few fibroblasts in tumor (e). In microphotographs a-e and g, arrowhead identifies CD34-positive blood vessels; black arrow in $g$ indicates lymphatic vessel. Microphotographs were taken at magnification (objective): $a-f: 20 x, g: 40 x$. 

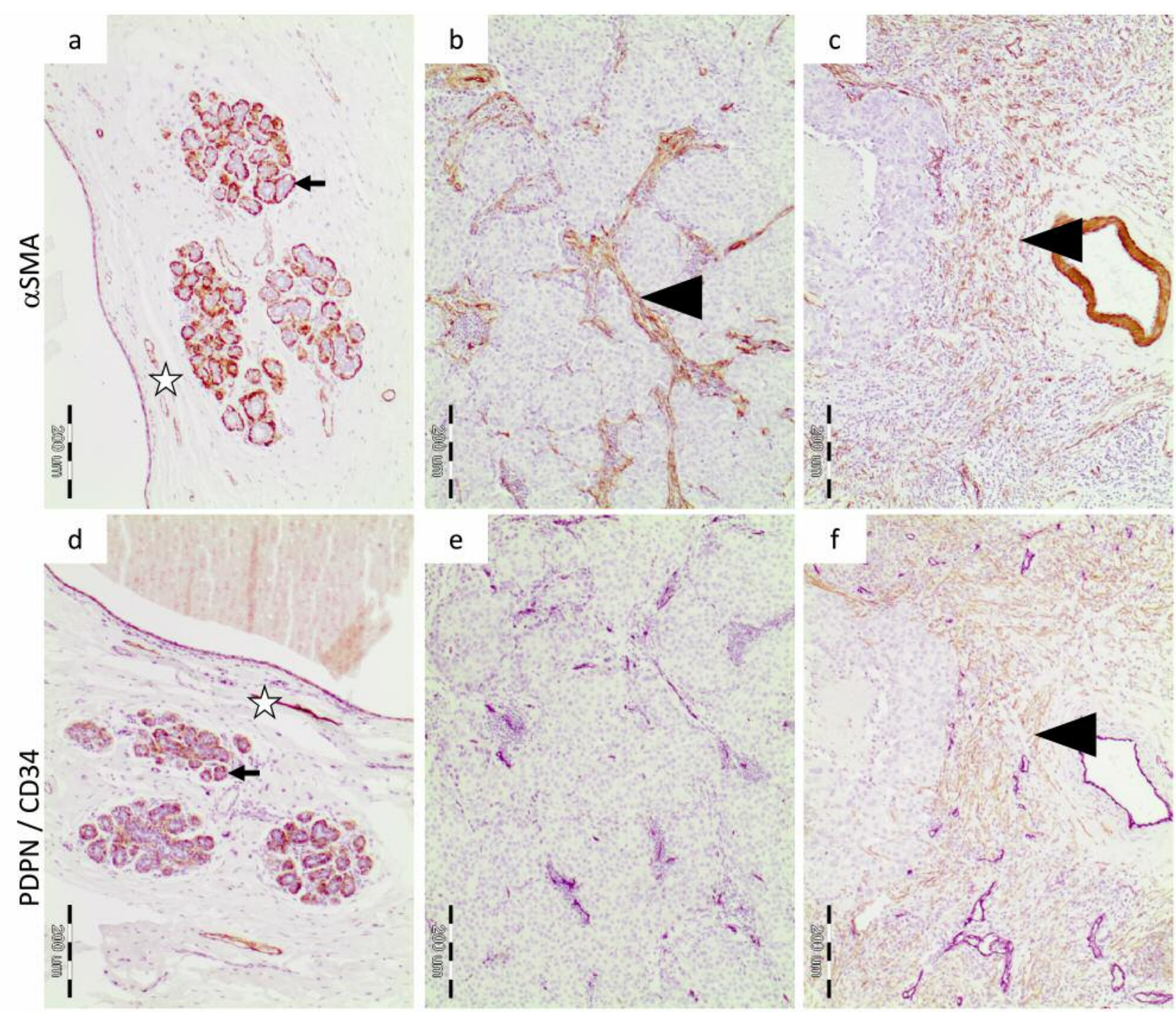

Figure 2. Expression of alpha smooth muscle actin ( $\alpha S M A)(a-c)$ and podoplanin (PDPN) (d-f) using double stain procedure with PDPN visualized with 3,3'-diaminobenzidine (DAB; brown color); and CD34 visualized with VECTOR VIP (violet color) from Peroxidase Substrate Kits (Vector Laboratories) in serial sections of normal breast tissue ( $a$ and $d$ ) and tumors (b-e and c-f). a: In normal breast tissue, expression of $\alpha$ SMA was found in myoepithelial cells of normal ducts and lobules (black arrow) and smooth muscle layer of blood vessels (white asterisk). $d$ : Expression of PDPN was observed in myoepithelial cells of normal ducts and lobules (black arrow) and epithelial cells of lymphatic vessels (white asterisk) (double stain). In one tumor $(b, e)$, cancer-associated fibroblasts were $\alpha \mathrm{SMA}^{+}\left(b\right.$, black arrowhead), and PDPN ${ }^{-}(e)$, while in the other (c-f), cancer-associated fibroblasts showed expression of both $\alpha S M A(c$, black arrowhead) and PDPN $(f$, black arrowhead).

lymph node involvement, as well as the possible prognostic significance of podoplanin expression in CAFs $(11,13,16$, 17). Correlation between the presence of ppCAFs and survival of trastuzumab-treated patients suggests that breast cancer stroma characteristics might influence sensitivity to trastuzumab-based adjuvant therapy.

It is suggested that early in tumorigenesis normal fibroblasts work against malignant progression. However, later on, their 'activation' takes place (which is similar to the process of wound healing) and upon it, fibroblasts turn on expression of $\alpha$ SMA. Such fibroblasts are referred to as CAFs and are related to promotion of tumor growth and invasiveness (4). This is confirmed by our observations: we observed aSMA immunonegativity in stroma of normal breast, and its immunopositivity in stromal CAFs. Additionally, we found that not all $\alpha \mathrm{SMA}^{+}$fibroblasts were additionally podoplanin ${ }^{+}$, which suggests that ppCAFs constitute only a subpopulation of all CAFs. 

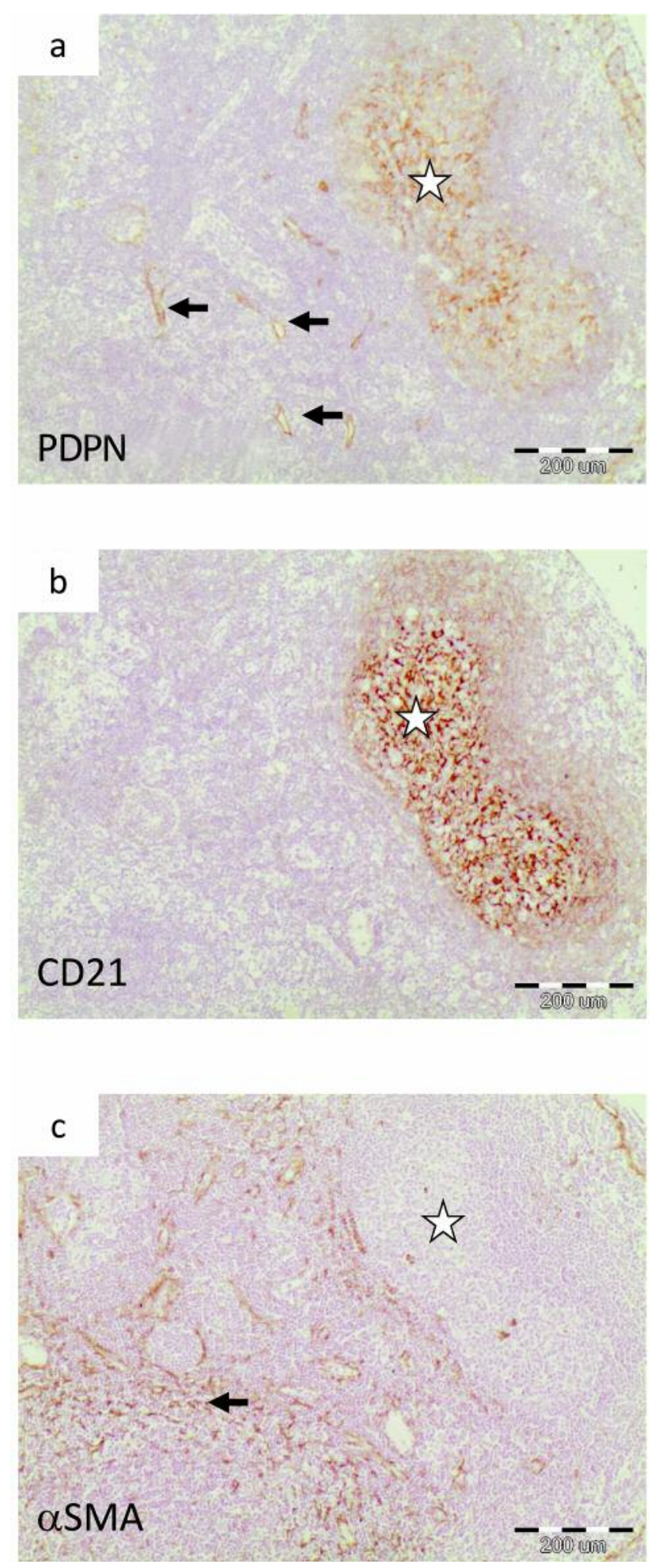

Figure 3. Nodal expression of: a: podoplanin (PDPN) - immunopositivity in lymphatic sinuses (black arrow) and follicular dendritic cells (FDCs; white asterisk); b: CD21 - immunopositivity in FDCs (white asterisk) and immunonegativity in fibroblastic reticular cells (FRC); c: alpha smooth muscle actin (aSMA) - immunonegativity in FDCs (white asterisk), immunopositivity in fibroblastic reticular cells (black arrow).
The main concern which was taken into account in our study was the comparison of stromal characteristics in primary tumor and synchronous LNMs, which might be important for planning future therapies targeted at stromal components of cancer (19). Our results revealed that podoplanin expression in CAFs was comparable in primary tumor and synchronous paired LNMs. Moreover, we did not observe the appearance of stromal ppCAFs in lymph node metastases from tumors lacking ppCAFs. This remains in line with previous studies, in which no significant changes between tumor and LNMs concerning fibroblast immunopositivity for ras homolog family member A (RHOA), Rac family small GTPase 1 (RAC1) (20), aSMA, S100 calcium binding protein A4 (S100A4) and vimentin (21) were observed. Both our own and the above-mentioned results speak in favor of the hypothesis (formulated based on animal model) suggesting that stromal components of primary tumor (the soil) could be brought by metastatic cells (the seed) and could influence the process of metastasis formation (1-3). To the best of our knowledge, our report is the first to compare expression of podoplanin in CAFs of primary tumor and synchronous LNMs.

An alternative to the above-mentioned hypothesis is one according to which stromal ppCAFs in LNMs are resident lymph node cells (25) such as podoplanin-positive FDCs $\left(\right.$ podoplanin $\left.^{+} / \alpha \mathrm{SMA}^{-} / \mathrm{CD} 21^{+} / \mathrm{CD}^{2} 3^{+}\right) \quad(22, \quad 23), \quad$ FRCs (podoplanin ${ }^{-} / \alpha \mathrm{SMA}^{+} / \mathrm{CD}^{2} 1^{-}$) (24), pericytes, endothelial cells, and adipocytes. However, based on immunohistochemistry we stated that ppCAFs (podoplanin ${ }^{+} / \alpha \mathrm{SMA}^{+}$ $/ \mathrm{CD} 21^{-}$) are: not FDCs because unlike FDCs they are $\alpha \mathrm{SMA}^{+}$ and $\mathrm{CD}_{2} 1^{-}$; not FRCs, because unlike FRCs, they are podoplanin $^{+}$; not pericytes because the latter do not express podoplanin; not endothelial cells, which do not show $\alpha$ SMA expression; and are not adipocytes which are podoplanin- and $\alpha$ SMA-negative. Obviously, we cannot exclude the possibility that the immunophenotype of the above-mentioned resident cells may change. Another hypothesis which could not be excluded by our study is that ppCAFs might originate from bone marrow-derived cells (25).

Contrary to the similarity in fibroblast immunophenotype in primary tumors and their synchronous LNMs, Kim et al. reported distant breast cancer metastasis (to liver, lungs, brain and bone) as presenting significant differences in expression of CAF-related proteins, particularly in podoplanin expression, which was found to be positive in primary tumor but negative in metastases (except for bone metastases) (9). In our study, the 'disappearance' of ppCAFs was observed in 23 cases. Moreover, we did not observe the appearance of podoplanin immunopositivity in LNMs from tumors without ppCAFs. Results of our work and others (9) suggest that metastatic cells tend to lose their own 'soil' (including ppCAFs) during their travel from the primary to a secondary site. Moreover, the longer the 'trip' is, the 
TUMOR

LNM

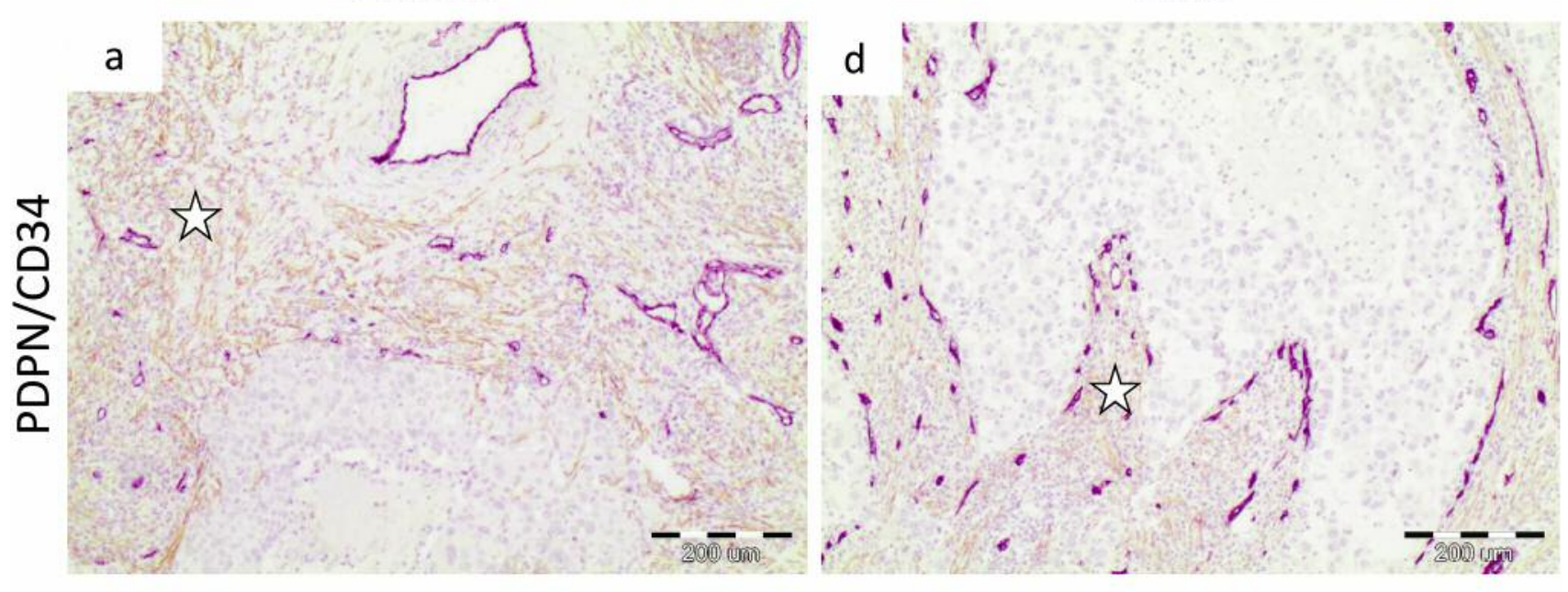

b

e
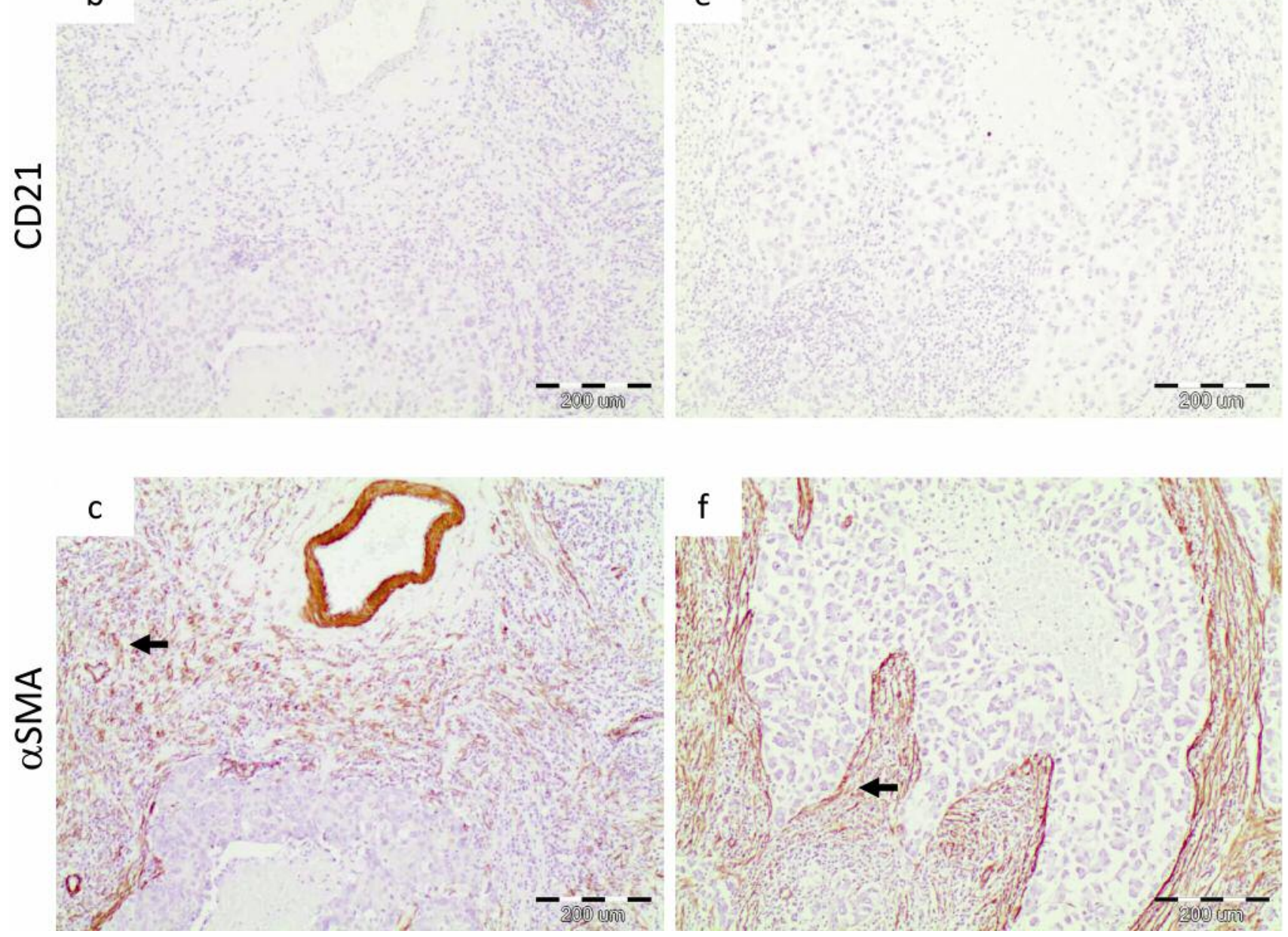

Figure 4. Expression of podoplanin (PDPN), CD34, CD21 and alpha smooth muscle actin ( $2 S M A)$ on serial sections of the same microscopic field of tumor (a-c) and paired synchronous lymph node metastases (d-f). Podoplanin: Immunopositive cancer-associated fibroblasts (CAFs) in stroma of primary tumor (white asterisk) (a) and paired lymph node metastases (white asterisk) (d). CD21: Immunonegativity of CAFs both in primary tumor (b) and paired lymph node metastases (e). aSMA-positive CAFs in stroma of primary tumor (arrow) (c) and paired lymph node metastases (arrow) (f). 
greater the amount of soil that is lost. Additionally, Kim et al. reported that only bone metastases presented elevated expression of stromal podoplanin (9). Therefore, it might be speculated that changes in the presence of ppCAFs (in tumor $v s$. LNM) might result in specific localization of distant metastases.

\section{Conclusion}

The immunophenotype of ppCAFs and the fact they are present (most frequently) both in tumor and in their corresponding LNMs speaks in favor of the hypothesis that ppCAFs might be brought by metastatic cells from the primary tumor (rather than being resident in lymph nodes). The presence of ppCAFs in the primary tumor might be an adverse prognostic factor in patients with HER2-positive breast cancer treated with trastuzumab in an adjuvant setting.

\section{Acknowledgements}

This study was financed by the National Science Centre based on decision number DEC-2013/09/B/NZ5/00764.

\section{References}

1. Duda DG, Duyverman AM, Kohno M, Snuderl M, Steller EJ, Fukumura D and Jain RK: Malignant cells facilitate lung metastasis by bringing their own soil. Proc Natl Acad Sci 107(50): 21677-21682, 2010.

2 Labernadie A, Kato T, Brugués A, Serra-Picamal X, Derzsi S, Arwert E, Weston A, González-Tarragó V, Elosegui-Artola A, Albertazzi L, Alcaraz J, Roca-Cusachs P, Sahai E and Trepat X: A mechanically active heterotypic E-cadherin/N-cadherin adhesion enables fibroblasts to drive cancer cell invasion. Nat Cell Biol 19: 224-237, 2017.

3 Ao Z, Shah SH, Machlin LM, Parajuli R, Miller PC, Rawal S, Williams AJ, Cote RJ, Lippman ME, Datar RH and El-Ashry D: Identification of cancer-associated fibroblasts in circulating blood from patients with metastatic breast cancer. Cancer Res 75: 4681-4687, 2015.

4 Marsh T, Pietras K and Allister SS: Fibroblasts as architects of cancer pathogenesis. Biochim Biophys Acta 1832(7): 1070-1078, 2013.

5 de Kruijf EM, van Nes JG, van de Velde CJ, Putter H, Smit VT, Liefers GJ, Kuppen PJ, Tollenaar RA and Mesker WE: Tumorstroma ratio in the primary tumor is a prognostic factor in early breast cancer patients, especially in triple-negative carcinoma patients. Breast Cancer Res Treat 125: 687-696, 2011.

6 Moorman AM, Vink R, Heijmans HJ, van der Palen J and Kouwenhoven EA: The prognostic value of tumour-stroma ratio in triple-negative breast cancer. Eur J Surg Oncol 38: 307-313, 2012.

7 Dekker TJ, van de Velde CJ, van Pelt GW, Kroep JR, Julien JP, Smit VT, Tollenaar RA and Mesker WE: Prognostic significance of the tumor-stroma ratio: validation study in node-negative premenopausal breast cancer patients from the EORTC perioperative chemotherapy (POP) trial (10854). Breast Cancer Res Treat 139: 371-379, 2013.
8 Park SY, Kim HM and Koo JS: Differential expression of cancer-associated fibroblast-related proteins according to molecular subtype and stromal histology in breast cancer. Breast Cancer Res Treat 149: 727-741, 2015.

9 Kim HM, Jung WH and Koo JS: Expression of cancerassociated fibroblast related proteins in metastatic breast cancer: an immunohistochemical analysis. J Transl Med 13: 222, 2015.

10 Ahn S, Cho J, Sung J, Lee JE, Nam SJ, Kim KM and Cho EY: The prognostic significance of tumor-associated stroma in invasive breast carcinoma. Tumor Biol 33: 1573-1580, 2012.

11 Pula B, Wojnar A, Werynska B, Ambicka A, Kruczak A, Witkiewicz W, Ugorski M, Podhorska-Okolow M and Dziegiel P: Impact of different tumour stroma assessment methods regarding podoplanin expression on clinical outcome in patients with invasive ductal breast carcinoma. Anticancer Res 33: 14471455, 2013.

12 Pula B, Jethon A, Piotrowska A, Gomulkiewicz A, Owczarek T, Calik J, Wojnar A, Witkiewicz W, Rys J, Ugorski M, Dziegiel P and Podhorska-Okolow M: Podoplanin expression by cancerassociated fibroblasts predicts poor outcome in invasive ductal breast carcinoma. Histopathology 59: 1249-1260, 2011.

13 Schoppmann SF, Berghoff A, Dinhof C, Jakesz R, Gnant M, Dubsky P, Jesch B, Heinzl $\mathrm{H}$ and Birner P: Podoplaninexpressing cancer-associated fibroblasts are associated with poor prognosis in invasive breast cancer. Breast Cancer Res Treat 134: 237-244, 2012.

14 Paulsson J and Micke P: Prognostic relevance of cancerassociated fibroblasts in human cancer. Semin Cancer Biol 25: 61-68, 2014.

15 Folgueira MA, Maistro S, Katayama ML, Roela RA, Mundim FG, Nanogaki S, de Bock GH and Brentani MM: Markers of breast cancer stromal fibroblasts in the primary tumour site associated with lymph node metastasis: a systematic review including our case series. Biosci Rep 33: 921-929, 2013.

16 Pula B, Witkiewicz W, Dziegiel P and Podhorska-Okolow M: Significance of podoplanin expression in cancer-associated fibroblasts: a comprehensive review. Int J Oncol 42(6): 1849$1857,2013$.

17 Niemiec JA, Adamczyk A, Ambicka A, Mucha-Małecka A, M Wysocki W and Ryś J: Triple-negative, basal marker-expressing, and high-grade breast carcinomas are characterized by high lymphatic vessel density and the expression of podoplanin in stromal fibroblasts. Appl Immunohistochem Mol Morphol AIMM 22: 10-16, 2014.

18 Adamczyk A, Grela-Wojewoda A, Domagała-Haduch M, Ambicka A, Harazin-Lechowska A, Janecka A, Cedrych I, Majchrzyk K, Kruczak A, Ryś J and Niemiec J: Proteins involved in HER2 signalling pathway, their relations and influence on metastasis-free survival in HER2-positive breast cancer patients treated with trastuzumab in adjuvant setting. J Cancer 8: 131-139, 2017.

19 Kota J, Hancock J, Kwon J and Korc M: Pancreatic cancer: Stroma and its current and emerging targeted therapies. Cancer Lett 391: 38-49, 2017.

20 Rozenchan PB, Pasini FS, Roela RA, Katayama ML, Mundim FG, Brentani H, Lyra EC and Brentani MM: Specific upregulation of RHOA and RAC1 in cancer-associated fibroblasts found at primary tumor and lymph node metastatic sites in breast cancer. Tumour Biol 36: 9589-9597, 2015. 
21 Mundim FG, Pasini FS, Nonogaki S, Rocha RM, Soares FA, Brentani MM and Logullo AF: Breast carcinoma-associated fibroblasts share similar biomarker profiles in matched lymph node metastasis. Appl Immunohistochem Mol Morphol AIMM 24: 712-720, 2016.

22 Agaimy A, Michal M, Hadravsky L and Michal M: Follicular dendritic cell sarcoma: clinicopathologic study of 15 cases with emphasis on novel expression of MDM2, somatostatin receptor 2A, and PD-L1. Ann Diagn Pathol 23: 21-28, 2016.

23 Xie Q, Chen L, Fu K, Harter J, Young KH, Sunkara J, Novak D, Villanueva-Siles E and Ratech H: Podoplanin (d2-40): a new immunohistochemical marker for reactive follicular dendritic cells and follicular dendritic cell sarcomas. Int J Clin Exp Pathol 1: 276-284, 2008 .
24 Blonska M, Agarwal NK and Vega F: Shaping of the tumor microenvironment: Stromal cells and vessels. Semin Cancer Biol 34: 3-13, 2015

25 Räsänen K and Vaheri A: Activation of fibroblasts in cancer stroma. Exp Cell Res 316(17): 2713-2722, 2010.

Received January 29, 2018

Revised February 23, 2018

Accepted February 26, 2018 